\title{
Ecología trófica del pez Arothron meleagris (Tetraodontiformes: Tetraodontidae) en el arrecife de Los Frailes, Baja California Sur, México
}

\author{
Xchel G. Moreno, L. Andrés Abitia, Araceli Favila, Francisco J. Gutiérrez \& Devis S. Palacios \\ Centro Interdisciplinario de Ciencias Marinas (CICIMAR-IPN), Playa Palo de Santa Rita s/n Apartado postal $592 \mathrm{La}$ \\ Paz, CP 23096, Baja Califórnia Sur, México: xmoreno@ipn.mx
}

Recibido 24-I-2008. Corregido 11-VII-2008. Aceptado 12-VIII-2008.

\begin{abstract}
Trophic ecology of the fish Arothron meleagris (Tetraodontiformes: Tetraodontidae) from Los Frailes reef, Southern Baja California, Mexico. Monthly samples were taken from November 2004 to October 2005 in the Los Frailes reef, BCS, Mexico. The objective was to determine the feeding habits of the pufferfish, Arothron meleagris, as well as possible variations in feeding patterns due to size and/or sex. We captured 101 specimens, with all stomachs contained food. The index of relative importance (IRI) was used to determine the main items; which were non-identified organic matter (NIOM) (30.44\%), Echinometra vanbrunti (26.25\%), Porifera (12.63\%), Pocillopora spp. (11.84\%), Bryozoa (5.37\%) and Porites spp. (4.83\%). Levin's index indicated a low trophic niche breadth $(B i=0.12)$ and allowed us to determine that $A$. meleagris is a specialist predator with strong preferences towards certain food types. The Morisita-Horn index showed a high trophic overlap between sexes $(C \lambda=0.78)$, seasons (hot and cold $C \lambda=0.95$ ) and among sizes (small and medium $C \lambda=0.94$; small and large $C \lambda=0.74$; medium and large $C \lambda=0.83$ ). Rev. Biol. Trop. 57 (1-2): 113-123. Epub 2009 June 30 .
\end{abstract}

Key words: Arothron meleagris, reef fish, diet, tropical eastern Pacific, Mexico.

El Pacífico Oriental Tropical no presenta especies coralívoras nativas (Robertson et al. 2004), este grupo está representado por dos especies residentes de afinidad Indopacífica del género Arothron: A. hispidus y A. meleagris, con amplia distribución desde el Golfo de California hasta Ecuador, incluye las islas oceánicas (Goodson 1988, Thomson et al. 2000, Robertson y Allen 2002).

Arothron meleagris forma parte importante de la comunidad de peces debido a su dominancia espacio-temporal, habita las zonas someras preferentemente con arrecifes rocosos o de coral del Bajo Golfo de California y puede encontrarse en profundidades de hasta $90 \mathrm{~m}$ (Allen y Robertson 1994, Thomson et al. 2000). De acuerdo con Montes-Hernández (2006) es una de las 10 especies más dominantes en el arrecife de Los Frailes, el cual se encuentra dentro del perímetro del Arrecife de Cabo Pulmo, mismo que fue declarado como área marina protegida (Parque Marino) en 1995 y constituye una de las zonas de mayor riqueza de especies en el Golfo de California (Reyes-Bonilla 1997).

A. meleagris presenta dos fases de coloración, una negra y otra dorada, ambas muy apreciadas por los acuaristas internacionales y alcanza un alto valor económico (AlmenaraRoldán 2000). Es una especie de hábitos diurnos, de nado lento y puede inflar su cuerpo por la ingesta de agua o aire, adaptación que permite disuadir a sus depredadores, también producen en sus tejidos un veneno muy potente "tetrodotoxina", el cual acumulan especialmente en el hígado, gónadas, ovarios e intestinos. La toxicidad varía dependiendo del área geográfica y de la época del año, además están equipados con numerosas glándulas celulares en la piel que secretan un mucus tóxico (Goodson 1988, Allen y Robertson 1994). 
La biología básica de $A$. meleagris ha sido poco estudiada y los antecedentes de trabajos en el Pacífico oriental tropical son específicamente investigaciones que solo evaluan cambios en la dieta de la especie de acuerdo a la disponibilidad de alimento (Hiatt y Strasburg 1960, Hobson 1974, Guzmán y Robertson 1989, Guzmán y López 1991, Reyes-Bonilla y Calderón-López 1999), evidenciándose la falta de estudios en donde se efectúen muestreos sistemáticos que aborden cambios en el espectro trófico de acuerdo con la temporalidad, desarrollo ontogénico y por sexos. En cuanto a su estrategia alimenticia Allen y Robertson (1994) reportaron que $A$. meleagris, hace uso de sus placas dentales en forma de pico de loro para cortar las porciones de corales, algas calcáreas y estructuras duras de invertebrados de los que se alimenta.

A pesar de la importancia ecológica y económica de A. meleagris en el Bajo Golfo de California, es reducida la información concerniente a la composición de la dieta y los hábitos alimenticios. Por lo que objetivo de este estudio fue analizar los hábitos alimenticios de A. meleagris, con la finalidad de caracterizar su espectro trófico y determinar sus posibles variaciones estacionales, talla y sexo, en el arrecife de Los Frailes B.C.S., México.

\section{MATERIAL Y MÉTODOS}

Se realizaron muestreos mensualmente durante noviembre 2004-octubre 2005, en el arrecife rocoso de Los Frailes, el cual se localiza en la parte sur de la Península de Baja California (entre los $23^{\circ} 5^{\prime} \mathrm{N}$ y los $109^{\circ}$ 5 , W). El área del arrecife mide $0.070 \mathrm{~km}^{2} \mathrm{y}$ en él se observan acumulaciónes de rocas de diferentes tamaños (desde canto rodado menor de $0.3 \mathrm{~m}$ hasta más de $3 \mathrm{~m}$ de longitud), que proporcionan un numero alto de oquedades, que van desde la zona intermareal hasta los 10 $\mathrm{m}$ de profundidad, con presencia de parches de arenosos y escasas formaciones coralinas. Las aguas de la zona son claras y la precipitación es escasa, siendo la temporada de lluvias de julio a septiembre (Anaya-Reyna, 1993; MontesHernández 2006).
La captura de los organismos se realizó con equipo de buceo básico y hawaiana entre las 10:00 y las 16:00 horas cuando la iluminación es mejor, en cada localidad de muestreo además se registró la temperatura del agua. Para cada ejemplar se registró la longitud total (LT), sexo y se extrajo su estómago. El contenido estomacal fue guardado en bolsas de plástico previamente etiquetadas, las cuales fueron congeladas para su posterior análisis en el laboratorio de ecología de peces del Centro Interdisciplinario de Ciencias Marinas (CICIMAR-IPN). Durante el análisis del contenido estomacal se procedió a separar los diferentes ítems de acuerdo al grupo taxonómico, identificándose hasta el mínimo taxon posible, dependiendo de su estado de digestión.

Para la identificación taxonómica de crustáceos y moluscos se utilizaron las claves de Brusca (1980) y Morris et al. (1980); para algas las claves de Abbott y Hollenberg (1976). Asimismo se contó con el apoyo de investigadores especialistas de CICIMAR y de la Universidad Autónoma de Baja California Sur (UABCS), los cuales corroboraron la identificación realizada.

Se calculó el porcentaje de frecuencia de aparición $(\% \mathrm{FA})$, porcentaje numérico $(\% \mathrm{~N})$ y porcentaje gravimétrico $(\% \mathrm{P})$. Una vez obtenidos estos valores, se calculó el índice de importancia relativa (IIR) (Pinkas et al. 1971), el cual incorpora los métodos anteriores por medio de la siguiente fórmula:

$$
\mathrm{IIR}=(\% \mathrm{P}+\% \mathrm{~N}) * \% \mathrm{FA}
$$

Se obtuvo la proporción sexual, dividiendo el número total de hembras entre el número total de machos; expresando la proporción de sexos con un numero 1 a la izquierda, que corresponde a un macho y a la derecha la proporción de hembras por macho. Para determinar si existian diferencias significativas entre las proporciones se utilizó el estadístico de prueba Chi-cuadrada (Zar 1999). Para la determinación de los intervalos de talla se emplearon los valores de longitud total y para determinar el número de intervalos se usó la fórmula de Sturges. Una 
vez obtenido el número de intervalos de clase se procedió a determinar su amplitud todo esto de acuerdo con Daniel (1997).

Se calculó la amplitud de la dieta $(B i)$ con el índice estandarizado de Levin (Hurlbert 1978); el cual asume valores de 0 a 1 . Cuando los valores de $B i$ son menores de 0.60 , el organismo se considera especialista, lo que indica que utiliza un número bajo de recursos y presenta una preferencia por ciertos alimentos. Cuando los valores son cercanos a uno $(>0.60)$, su espectro es generalista, es decir, utiliza todos los recursos sin ninguna selección.

Para evaluar el traslape de dietas entre tallas, sexos y temporada se aplicó el índice de Morisita-Horn (Smith y Zaret 1982). Este índice varía entre cero (cuando las dietas son completamente distintas) y uno (cuando las dietas son similares). Se considera un traslape biológico significativo cuando el valor excede a 0.60 ; y valores menores a 0.60 se considera que no hay traslape (Langton 1982). Asimismo con la finalidad de corroborar si existían diferencias significativas entre los resultados en el análisis del traslape trófico, se realizó la prueba no paramétrica de Kruskal-Wallis (Zar 1999).

\section{RESULTADOS}

Se capturaron un total de 101 ejemplares del tamboril de oro A. meleagris, todos los estómagos contenían alimento. Se identificaron un total de 34 ítems: 12 correspondieron a algas, seis gasterópodos, dos corales, dos bivalvos, dos erizos, dos langostas, dos pelecípodos, un Bryozoa, una estrella de mar, una esponja, un poliqueto, un balano y materia orgánica no identificada (MONI) (Cuadro 1).

Los ítems en los estómagos registraron una biomasa total de 2225.55 g, los más importantes de acuerdo al método gravimétrico fueron el erizo Echinometra vanbrunti (24.70\%) y el coral Pocillopora spp. (14.71 \%). Cabe destacar que la MONI registró el valor más alto de la biomasa $(25.55 \%)$.

Con el método numérico se cuantificaron un total de 133 ítems, destacando las esponjas (Porifera) con un $22.66 \%$, seguidos por el molusco gasterópodo Crepidula arenata con el $19.54 \%$, y el invertebrado Bryozoa con el 15.03 $\%$. Los ítems más frecuentes fueron el erizo $E$. vanbrunti (65.34\%) y el coral Pocillopora spp. (49.50 \%). La MONI se registro en el $73.26 \%$ de los estómagos analizados.

Al incorporar los valores porcentuales de los métodos anteriores en el índice de importancia relativa (IIR), tomando solo los valores que sumaran más de $90 \%$ se determinó que los ítems más importantes del espectro trófico de A. meleagris fueron: E. vanbrunti $(26.25 \%)$, Porifera (12.63\%), Pocillopora spp. (11.84\%), Bryozoa $(5.37 \%)$, Porites spp. (4.83\%) y $C$. arenata $(3.82 \%)$. Cabe destacar que la MONI ocupo el segundo lugar de la importancia relativa con el $25.55 \%$ (Fig. 1).

Para las hembras se registraron 28 estómagos con alimento, a partir de los cuales, los ítems más importantes fueron: E. vanbrunti (25.17\%), Pocillopora spp. (11.59\%), Porifera (9.07 \%), Porites spp. (8.43 \%), Bivalvos (4.46\%), Bryozoa (4.25\%), C. arenata (3.57 $\%)$ y Notoacmea fascicularis (2.92\%). Para los machos se registraron 68 estómagos con alimento, se encontró que los ítems más importantes del espectro trófico fueron; $E$. vanbrunti (24.71\%), Pocillopora spp. (16.76\%), Porites spp. (7.72\%) y Porifera (3.66). Se presentaron 5 ejemplares de $A$. meleagris con los estómagos con alimento, a los cuales no fue posible sexar por su incipiente desarrollo gonádico. Tanto en la dieta de hembras y machos la MONI registro valores de IIR superiores al 22\% (Fig. 2). Se determinó que la proporción de machos y hembras fue significativamente diferente de 1:1 $\left(X^{2}=\mathrm{p}>0.05\right)$, la proporción fue de 1:0.41.

Para el análisis de tallas se definieron como tallas chicas los organismos del intervalo de 9-15 cm, obteniéndose 23 estómagos con alimento, siendo los ítems más importantes: E. vanbrunti (38.01\%), Pocillopora spp. (10.31\%), Amphiroa beauvosii (8.45\%), alga no identificada (2.93\%), Bryozoa (2.36\%). Las tallas medianas correspondieron al intervalo de $16-22 \mathrm{~cm}$, de los que se analizaron 50 estómagos con alimento los ítems más importantes fueron E. vanbrunti (28.54\%), Pocillopora 
CUADRO 1

Espectro trófico de Arothron meleagris en el arrecife de Los Frailes

TABLE 1

Trophic spectrum of the Arothron meleagris in Los Frailes reef

\begin{tabular}{|c|c|c|c|c|c|c|c|c|}
\hline Organismo & FA & $\% \mathrm{FA}$ & $\mathrm{N}$ & $\% \mathrm{~N}$ & $\mathrm{P}$ & $\% \mathrm{P}$ & IIR & $\%$ IIR \\
\hline Alga no identificada & 38 & 37,62 & 0 & 0 & 4,61 & 0,21 & 7,79 & 0,13 \\
\hline \multicolumn{9}{|l|}{ División Chlorophyta } \\
\hline Cladophora spp. & 9 & 8,91 & 0 & 0 & 1,3 & 0,06 & 0,52 & 0,01 \\
\hline Ulva lactuca & 29 & 28,71 & 0 & 0 & 1,85 & 0,08 & 2,39 & 0,04 \\
\hline \multicolumn{9}{|l|}{ División Phaeophyta } \\
\hline Dictyopteris undulata & 13 & 12,87 & 0 & 0 & 3,4 & 0,15 & 1,97 & 0,03 \\
\hline Dictyota dichotoma & 23 & 22,77 & 0 & 0 & 1,65 & 0,07 & 1,69 & 0,03 \\
\hline Gracilaria spp. & 11 & 10,89 & 0 & 0 & 0,91 & 0,04 & 0,45 & 0,01 \\
\hline Padina durvillaei & 32 & 31,68 & 0 & 0 & 13,46 & 0,6 & $19, .16$ & 0,31 \\
\hline \multicolumn{9}{|l|}{ División Rhodophyta } \\
\hline Amphiroa beauvosii & 49 & 47,52 & 0 & 0 & 16,03 & 0,72 & 34,22 & 0,56 \\
\hline Ceramium flaccidum & 13 & 12,87 & 0 & 0 & 1,77 & 0,08 & 1,02 & 0,02 \\
\hline Hypnea spp. & 38 & 37,62 & 0 & 0 & 5,55 & 0,25 & 9,39 & 0,15 \\
\hline Jania adherens & 17 & 16,83 & 0 & 0 & 2,45 & 0,11 & 1,86 & 0,03 \\
\hline Polysiphonia pacifica & 23 & 22,77 & 0 & 0 & 6,23 & 0,28 & 6,37 & 0,1 \\
\hline \multicolumn{9}{|l|}{ Phylum Cnidaria } \\
\hline \multicolumn{9}{|l|}{ Pocilloporidae } \\
\hline Pocillopora spp. & 50 & 49,5 & 0 & 0 & 327,51 & 14,72 & 728,51 & 11,85 \\
\hline \multicolumn{9}{|l|}{ Poritidae } \\
\hline Porites spp. & 27 & 26,73 & 0 & 0 & 247,31 & 11,11 & 297,06 & 4,83 \\
\hline \multicolumn{9}{|l|}{ Phylum Crustacea } \\
\hline \multicolumn{9}{|l|}{ Palinuridae } \\
\hline Panulirus inflatus & 8 & 7,92 & 0 & 0 & 31,46 & 1,41 & 11,2 & 0,18 \\
\hline Panulirus interruptus & 1 & 0,99 & 0 & 0 & 34,68 & 1,56 & 1,54 & 0,03 \\
\hline \multicolumn{9}{|l|}{ Balanidae } \\
\hline Balanus spp. & 1 & 0,99 & 7 & 5,26 & 3,15 & 0,14 & 5,35 & 0,09 \\
\hline \multicolumn{9}{|l|}{ Phylum Mollusca } \\
\hline Clase Gastropoda & 1 & 0,99 & 2 & 1,5 & 4,81 & 0,22 & 1,7 & 0,03 \\
\hline Notoacmea fascicularis & 4 & 3,96 & 8 & 6,02 & 9,9 & 0,44 & 25,58 & 0,42 \\
\hline Phenacolepas malonei & 4 & 3,96 & 4 & 3,01 & 3,7 & 0,17 & 12,57 & 0,2 \\
\hline \multicolumn{9}{|l|}{ Hipponicidae } \\
\hline Hipponix panamensis & 3 & 2,97 & 6 & 4,51 & 39,68 & 1,78 & 18,7 & 0,3 \\
\hline \multicolumn{9}{|l|}{ Calyptraeacea } \\
\hline Crepidula aculeata & 2 & 1,98 & 4 & 3,01 & 12,02 & 0,54 & 7,03 & 0,11 \\
\hline Crepidula arenata & 11 & 10,89 & 26 & 19,55 & 45,28 & 2,03 & 235,07 & 3,82 \\
\hline Ostrea fisheri & 1 & 0,99 & 1 & 0,75 & 1,06 & 0,05 & 0,79 & 0,01 \\
\hline Clase Bivalvia & 1 & 0,99 & 5 & 3,76 & 2,5 & 0,11 & 3,83 & 0,06 \\
\hline \multicolumn{9}{|l|}{ Tellinidae } \\
\hline Tellina spp. & 8 & 7,92 & 14 & 10,53 & 43,52 & 1,96 & 98,86 & 1,61 \\
\hline
\end{tabular}


CUADRO 1 (Continuación)

Espectro trófico de Arothron meleagris en el arrecife de Los Frailes

TABLE 1 (Continued)

Trophic spectrum of the Arothron meleagris in Los Frailes reef

\begin{tabular}{|c|c|c|c|c|c|c|c|c|}
\hline Organismo & FA & $\% \mathrm{FA}$ & $\mathrm{N}$ & $\% \mathrm{~N}$ & $\mathrm{P}$ & $\% \mathrm{P}$ & IIR & $\%$ IIR \\
\hline \multicolumn{9}{|l|}{ Phylum Echinodermata } \\
\hline \multicolumn{9}{|l|}{ Cidaridae } \\
\hline Eucidaris thouarssii & 1 & 0,99 & 0 & 0 & 24,43 & 1,1 & 1,09 & 0,02 \\
\hline \multicolumn{9}{|l|}{ Echinometridae } \\
\hline Echinometra vanbrunti & 66 & 65,35 & 0 & 0 & 549,86 & 24,71 & 1614,49 & 26,26 \\
\hline \multicolumn{9}{|l|}{ Asteroidea } \\
\hline Phanataria unifacialis & 1 & 0,99 & 1 & 0,75 & 21,96 & 0,99 & 1,72 & 0,03 \\
\hline \multicolumn{9}{|l|}{ Orden Scleractinia } \\
\hline MONI & 74 & 73,27 & 0 & 0 & 568,66 & 25,55 & 1872,07 & 30,45 \\
\hline \multicolumn{9}{|l|}{ Annelida } \\
\hline Polichaeta & 1 & 0,99 & 0 & 0 & 20,6 & 0,93 & 0,92 & 0,01 \\
\hline Bryozoa & 20 & 18,81 & 20 & 15,04 & 56,1 & 2,52 & 330,3 & 5,37 \\
\hline Phylum Porifera & 30 & 29,7 & 30 & 22,56 & 75,29 & 3,38 & 770,48 & 12,53 \\
\hline TOTALES & 101 & 605,94 & 133 & 100 & 2225,56 & 100 & 6148,2 & 100 \\
\hline
\end{tabular}

Valores absolutos y porcentuales de los métodos de frecuencia de aparición (FA), numérico $(\mathrm{N})$, gravimétrico (P) e índice de importancia relativa (IIR).

Values and percentages based on frequency of occurrence (FA), number $(\mathrm{N})$, Weight $(\mathrm{P})$ and Index of relative impoprtance (IIR).

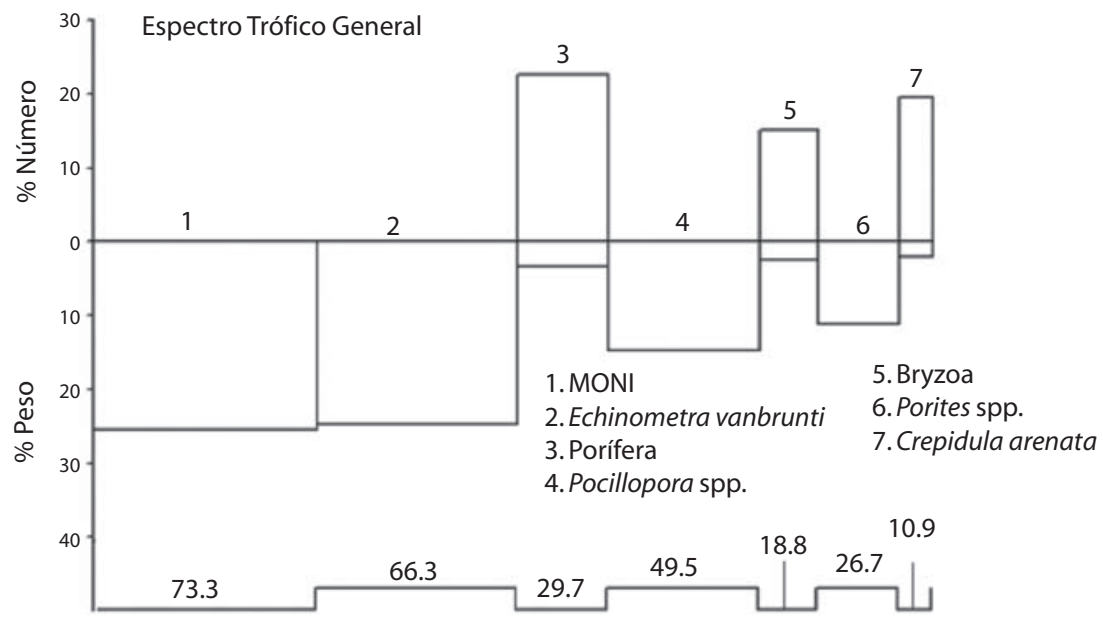

\% Frecuencia de Aparición

Fig. 1. Espectro trófico (IIR) de A. meleagris presentado como porcentajes de número, peso y frecuencia de aparición.

Fig. 1. Trophic spectrum (IRI) of the guineafowl puffer, A. meleagris, shown as percent number, weight and frequency of occurrence. 


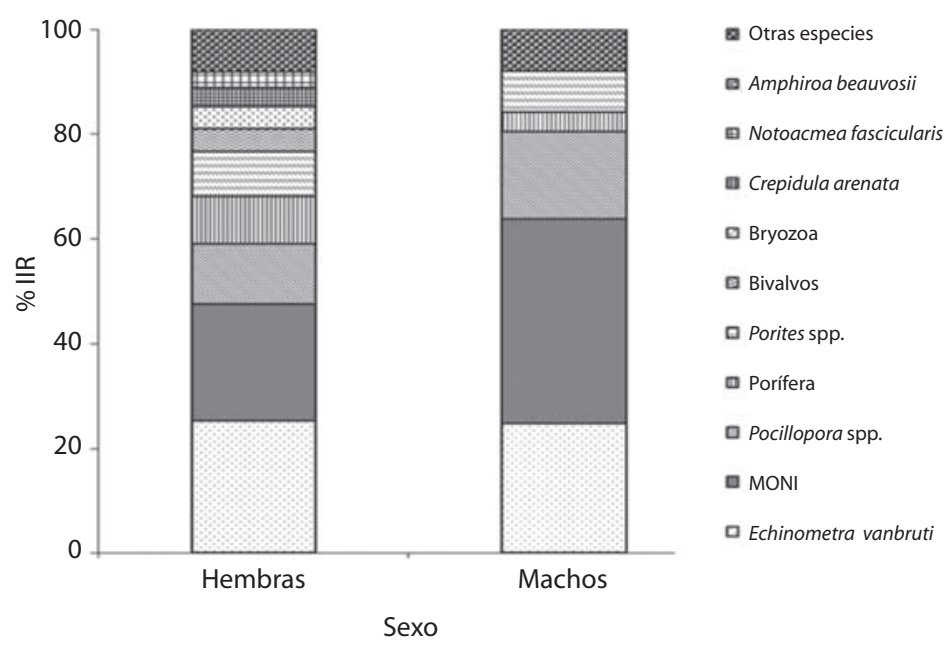

Fig. 2. Espectro trófico de A. meleagris por hembras y machos.

Fig. 2. Trophic spectrum of $A$. meleagris for females and males.

spp. (11.21\%), Porites spp. (8.40\%), A. beauvosii $(7.17 \%)$, Hypnea spp. (5.90\%), alga no identificada (4\%). Dentro de las tallas grandes se agruparon a los organismos de $23-32 \mathrm{~cm}$, de los cuales se revisaron 28 estómagos con alimento, en donde Pocillopora spp. (15.12\%), E. vanbrunti (14.68\%), Porites spp. (12.28\%) y Porífera $(7.73 \%)$ fueron los ítems principales. Se debe señalar que la materia organica no identificada en todas las tallas registró un valor alto de la importancia relativa. (Fig. 3)

Para el análisis estacional de los meses muestreados fueron divididos en dos temporadas del año de acuerdo con Álvarez-Borrego y Schwartzlose (1979) y considerando los registros de temperatura tomados en el sitio de muestreo, noviembre-diciembre 2004 y febrero-abril 2005 considerados como temporada fría (22-25 ${ }^{\circ} \mathrm{C}$ ), mientras que de junio-octubre del 2005 estimados como temporada cálida $\left(26-31^{\circ} \mathrm{C}\right)$. Para la temporada fría, $A$. meleagris se alimento de E. vanbrunti (36,02\%), Pocillopora spp. (10.48\%) y Porites spp. (9.03\%), conformando más del 55\% del índice de importancia relativa en un total de 55 estómagos llenos. Para la época cálida los ítems más importantes fueron, E. vanbrunti (26,74\%), Pocillopora spp. (17.83
\%), Porifera (6.41\%) y Porites spp. (3.32) con un total de 46 estómagos. La MONI en la temporada fría registro $35.99 \%$ y en la temporada cálida 36.39\% del IIR (Fig. 4).

Para la amplitud y el traslape de las dietas, debido a la omnivoría de esta especie, no se pudo tomar en cuenta los valores absolutos del método numérico, ya que en el caso de las algas y corales identificados, no fue posible la cuantificación de unidades, por lo que se optó por el uso de los valores absolutos del índice de importancia relativa. Al aplicar el índice de Levin se observó que la amplitud de la dieta (Bi), A. meleagris se puede caracterizar como especialista ya que el valor estimado de amplitud fue muy bajo $(B i=0.12)$ en el espectro trófico general. Por lo cual, se considera como un depredador, con marcadas preferencias hacia algunos ítems (E. vanbrunti, Pocillopora spp., Porites spp., Porifera, Bryozoa,. y C. arenata) (Cuadro 2). De acuerdo al índice de MorisitaHorn, se presentó un traslape entre las y dietas por sexo (hembras y machos $C \lambda=0.78$ ), entre tallas (Chica y mediana $C \lambda=0.94$, chica y grande $C \lambda=0.74$; mediana y grande $C \lambda=0.83)$ y temporadas (cálida y fría $C \lambda=0.95)$.(Cuadro 3). La prueba de Kruskal-Wallis corroboró que 


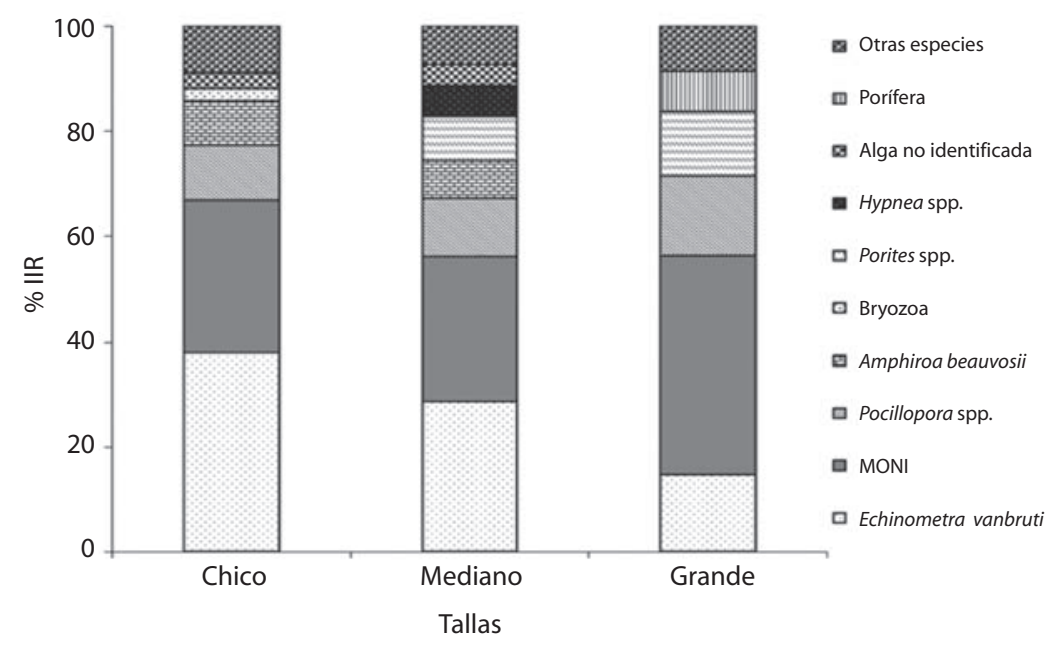

Fig. 3. Análisis trófico de A. meleagris por intervalo de talla, presentado como porcentajes del índice de importancia relativa.

Fig. 3. Trophic analysis of the A. meleagris by size interval, shown as percentages of the index of relative importance.

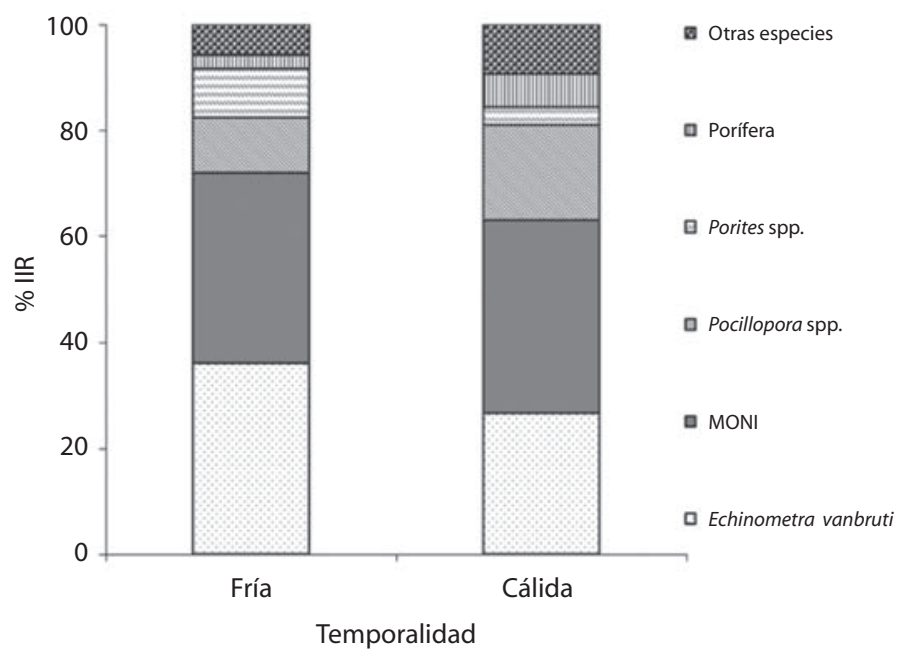

Fig. 4. Indice de importancia relativa estacional de A. meleagris.

Fig. 4. Seasonal index of relative importance for the A. meleagris. 
CUADRO 2

Amplitud de dieta de A. meleagris de acuerdo al el índice de Levin. General, por tallas, sexo y estacionalidad

TABLE 2

Diet breadth of A. meleagris according to Levin's index, general, by size, sex and season

$\begin{array}{lc}\quad \text { Categoría } & \text { Valor } \\ \text { General } & 0,12 \\ \text { Tallas } & \\ \text { Talla chica } & 0,16 \\ \text { Talla mediana } & 0,15 \\ \text { Talla grande } & 0,16 \\ \text { Sexo } & \\ \text { Hembra } & 0,16 \\ \text { Macho } & 0,13 \\ \text { Estacionalidad } & \\ \text { Época Cálida } & \\ \text { Época Fría } & 0,16\end{array}$

en todas las combinaciones no se registraron diferencias significativas $(\mathrm{p}<0.05)$.

\section{DISCUSIÓN}

El análisis del contenido estomacal en peces es importante para entender la ecología trófica y un medio para conocer las interacciones entre los depredadores y sus presas (Jackson et al. 2000). En la literatura se señala la gran presión depredatoria que Arothron meleagris ejerce sobre el coral, esponjas y equinodermos así como en algunas algas (Glynn et al. 1972, Guzmán y López 1991, Reyes-Bonilla y Calderón-Aguilera 1999), lo cual es respaldado por los resultados en el presente trabajo. Guzmán y López (1991) mencionan que A. meleagris se alimenta de algas coralinas, esponjas, equinodermos y otros invertebrados, y que tiene una marcada preferencia por el coral Pocillopora spp., el cual es el más abundante $y / o$ disponible en un arrecife colombiano. Asimismo, Glynn et al. (1972) describe que en un arrecife coralino de Panamá la dieta de $A$. meleagris (14 individuos con un intervalo de tallas de 13,1 a 18,0 cm.) en su mayoría está compuesta por coral con seis especies, algas calcáreas, bivalvos y ophiuroideos.

Reyes-Bonilla y Calderón-López (1999) reportaron que A. meleagris es un depredador generalista que puede alimentarse de algas, moluscos, crustáceos, coral y otras especies. Tambien estos autores determinaron que en el área de Cabo Pulmo, la cantidad total de carbonato de coral removido por A. meleagris es más pequeño que lo reportado para los arrecifes de Panamá.

CUADRO 3

Traslape trófico de A. meleagris de acuerdo al indice de Morisita-Horn, por tallas, sexo y estacionalidad.

TABLE 3

Trophic overlap of A. meleagris according to the Morisita-Horn index, by size, sex and season

\begin{tabular}{|c|c|c|c|}
\hline Tallas & Talla chica & Talla mediana & Talla grande \\
\hline Talla chica & 1 & 0,94 & 0,74 \\
\hline Talla mediana & & 1 & 0,83 \\
\hline Talla grande & & & 1 \\
\hline Sexo & Hembra & Macho & \\
\hline Hembra & 1 & 0,78 & \\
\hline Macho & & 1 & \\
\hline Estacionalidad & Época Cálida & Época Fría & \\
\hline Época Cálida & 1 & 0,95 & \\
\hline Época Fría & & 1 & \\
\hline
\end{tabular}


En el presente trabajo se analizaron 101 individuos de A. meleagris de un intervalo de tallas entre 9 a $32 \mathrm{~cm}$. Registrándose que su dieta se compone de; esponjas (Porifera), erizos (Echinometra vanbrunti), corales (Pocillopora spp., Porite spp.) y otras especies de invertebrados (Bryozoa, y Crepidula arenata). Lo cual coincide con los antecedentes, presentándose algunas diferencias en cuanto a la composición específica de la dieta, lo cual puede ser atribuible a las evidentes diferencias existentes entre los grados de exposición al oleaje, temperatura del agua, transparencia y escurrimientos de estos sistemas arrecifales. También se debe de señalar que las tallas registradas para $A$. meleagris en nuestro trabajo y el número de individuos son mayores a la de los trabajos de Glynn et al. (1972) y Guzmán y López (1991), lo cual pudiera también tener influencia en las diferencias registradas en la dieta de este depredador.

En los trabajos de Glynn et al. (1972) y Guzmán y López (1991) mencionan que $A$. meleagris es un organismo generalista, su dieta se basa principalmente en las especies más abundantes de coral y la complementa con organismos presentes en los sistemas arrecifales, en el presente trabajo no se encontró un comportamiento de este tipo, de acuerdo al índice de Levin, este pez, se puede caracterizar como un especialista, ya que aunque se alimenta de un variado número de ítems tiene una marcada preferencia por especies en particular, ya que la frecuencia de aparición en la dieta fue dominada por cinco ítems (erizos, corales, esponjas, moluscos y briozoos).

En el área de estudio se encuentran tres especies de peces; Diodon holocanthus, Suflamen verres y Melichthys niger que presentan un espectro trófico similar al de $A$. meleagris ya que estos organismos se alimentan de una gran variedad de invertebrados del bentos, además de ubicarse en un nivel trófico similar (Álvarez-Philip y Reyes-Bonilla 2006). Lo anterior pudiera implicar una posible competencia interespecífica, pero bajo las condiciones de repartición del recurso alimenticio. En la mayoría de los casos en donde se reporta una similitud de dietas, se da el efecto de la alta abundancia del alimento y en donde el recurso no es tan abundante entran en función otros factores como la segregación espacial y temporal, así como la plasticidad trófica de las especies (Cruz-Escalona et al. 2000).

Se registró un alto traslape de la dieta, lo cual evidencia que los organismos se alimentaron de presas en común, presentándose una muy pequeña variación en cuanto a la composición de las dietas por temporadas del año, tallas y sexos. Lo cual indica que no existe ningún tipo de segregación, ya que aparentemente los individuos de esta especie se encuentran agrupados y al momento de alimentarse consumen los mismos tipos presa, los organismos más grandes pueden fragmentar el alimento con mayor facilidad y así los individuos más pequeños pueden aprovechar, lo que a los otros se les escapa al momento de alimentarse.

El efecto que tienen los peces que incluyen en su dieta al coral, ha sido un tema de mucha controversia y de acuerdo con Reyes-Nivia et al. (2004) desde el viaje de C. Darwin en el Beagle a las Isla de Cocos-Keeling durante 1831-1836, se han mencionado las actividades erosivas de los peces y en especial de los pericos o loros (Scaridae), sobre el coral. En este sentido, tanto Glynn et al. (1972) como Guzmán y López (1991) mencionan que $A$. meleagris actúa como dispersador y regenerador de coral ya que al alimentarse de este lo fragmentan y por la acción del ramoneo permiten su dispersión y crecimiento, lo cual evidentemente ayuda al desarrollo de los sistemas coralinos.

La depredación de coral es un factor clave que controla en parte la organización de las comunidades arrecifales (Glynn et al. 1972) y de hecho ha sido reportada como una forma incesante de perturbación biológica. ReyesNivia et al. (2004), reportaron que en general la depredación de los corales por parte de los peces y en especial del pez perico Sparisoma viridae es un suceso habitual en estos arrecifes.

Reyes-Bonilla y Calderón-Aguilera (1999) citan que los organismos coralívoros no tienen un fuerte impacto sobre la estructura 
comunitaria de los corales en el área de Cabo Pulmo y concluyen que los factores bióticos (principalmente depredación) son menos importantes que los factores oceanográficos y abióticos en cuanto al control de la estructura de la comunidad arrecifal de la región del Pacífico oriental. Aunque el presente trabajo no se basó en el estudio de la acción de $A$. meleagris sobre el coral, puede existir la posibilidad de que debido a la gran abundancia de esta especie y su probada conducta trófica coralívora, este tipo de actividad de depredación pudiera tener algún tipo de impacto en el desarrollo de los corales presentes en el arrecife de los Frailes.

\section{AGRADECIMIENTOS}

Al Instituto Politécnico Nacional (IPN) por el apoyo recibido a través de la COFAA y EDI. Xchel G. Moreno Sánchez y Deivis S. Palacios Salgado agradecen al Consejo Nacional de Ciencia y Tecnología (CONACyT) y al Programa Institucional de Formación de Investigadores (PIFI-IPN). Finalmente nuestro agradecimiento a cuatro revisores anónimos que con sus comentarios mejoraron el trabajo.

\section{RESUMEN}

Con la finalidad de determinar los hábitos alimenticios del tamboril de oro Arothron meleagris, así como sus posibles variaciones de alimentación por talla y sexo, se realizaron muestreos mensuales de noviembre 2004 a octubre 2005, en el arrecife de Los Frailes, Baja California Sur, México. Se capturaron un total de 101 ejemplares, en los cuales todos los estómagos contenían alimento. Se aplicó el índice de importancia relativa (IIR) para determinar los principales categorías alimenticias (ítems), los cuales fueron: Echinometra vanbrunti (26.25\%), Porifera (12.63 \%), Pocillopora spp. (11.84\%), Bryozoa (5.37\%) y Porites spp. (4.83\%). El índice de Levin indica baja amplitud del nicho trófico $(B i=0.12)$ por lo que se le puede considerar como un depredador especialista con marcadas preferencias hacia algunos tipos alimentarios. El índice de MorisitaHorn indicó que existe un traslape alto entre las dietas por sexo (hembras y machos $C \lambda=0.78$ ), temporadas (cálida y fría $C \lambda=0.95$ ) y entre tallas (chica y mediana $C \lambda=0.94$, chica y grande $C \lambda=0.74$, mediana y grande $C \lambda=0.83$ ).

Palabras claves: Arothron meleagris, peces de arrecife, dieta, Pacífico oriental tropical, México.

\section{REFERENCIAS}

Abbott, I.A. \& G.J. Hollenberg. 1976. Marine Algae of California. Stanford University. Stanford, California, EEUU.

Allen, G. R. \& D.R. Robertson. 1994. Fishes of the tropical eastern Pacific. Honolulu: University of Hawaii, Hawaii, EEUU.

Almenara-Roldan, S.C. 2000. Demanda internacional de especies marinas ornamentales del Golfo de California, p. 39-47. En: O. Aburto-Oropeza \& C. Sánchez-Ortiz (eds). Recursos arrecifales del Golfo de California. Estrategias de manejo para las especies marinas de ornato. Universidad Autónoma de Baja California Sur y Brich Aquarium at SCRIPPS. $1^{\text {a }}$ edición, Baja California Sur, México.

Alvarez-Borrego, S \& R.A. Schwartzlose. 1979. Masas de agua del Golfo de California. Ciencias Marinas 6: 43-63.

Alvarez Filip, L. \& H. Reyes Bonilla. 2006. Comparison of community structure and functional diversity of fishes at Cabo Pulmo coral reef, B.C.S. Mexico between 1987 and 2003. Proceedings of 10th International Coral Reef Symposium. Okinawa, Japón..

Anaya-Reyna, G. 1993. Conservación del arrecife coralino de Cabo Pulmo: Avances sobre el proceso de planeación y propuesta de manejo. Tesis de Licenciatura. Universidad Autónoma de Baja California Sur, Baja California Sur, México.

Brusca, R. 1980. Common intertidal invertebrates of the Gulf of California. University Arizona. Tucson, Arizona, EEUU.

Cruz-Escalona, V.H., L.A. Abitia-Cardenas, L.Campos Davila \& F. Galvan-Magaña. 2000. Trophic interrelations of the three most abundant fish species from Laguna San Ignacio, Baja California Sur, Mexico. Bulletin Marine Science 66:361-373.

Daniel, W.W. 1997. Bioestadística. Base para el análisis de las ciencias de la salud. Limusa. México D.F., México.

Glynn, P.W., R.H. Stewart \& J.E. Mccosker. 1972. Pacific coral reefs of Panama: structure, distribution and predators. Geol. Rundschau. 61:483-519.

Goodson, G. 1988. Fishes of the Pacific Coast: Alaska to Perú, including the Gulf of California and the Galápagos Islands. Stanford University, Palo Alto, California, EEUU.

Guzmán, M.H \& R.D. Robertson. 1989. Population and feeding responses of the corallivorous pufferfish 
Arothron meleagris to coral mortality in the eastern Pacific. Marine Ecol. Progress Series 55:121-131.

Guzmán, M.H. \& J.D. López. 1991. Diet of the corallivorous pufferfish Arothron meleagris (Pisces: Tetraodontidae) at Gorgona Island, Colombia. Rev. Biol. Trop. 39:203-206.

Hiatt, R.W. \& D.C. Strasburg. 1960. Ecological relationships of the fish fauna on coral reefs of the Marsall Island. Ecol. Monographs 30: 65-127.

Hobson, E.S. 1974. Feeding relationships of teleostean fishes on coral reefs in Kona, Hawaii. Fishery Bulletin 72:915-1031.

Hurlbert, S.H. 1978. The measurement of niche overlap and some relatives. Ecology 59: 67-77.

Jackson, G.D., N.G. Buxton, \& M.J.A. George. 2000. Diet of the southern opah Lampris immaculatus on the Patagonoian Shelf; significance of the squid Morotheuthis ingens and anthropogenic plastic. Marine Ecol. Progress Series 206:261-271.

Langton, R.W. 1982. Diet overlap between the Atlantic cod Gadus Morhua, silver hake Merluccius bilinearis and fifteen other northwest Atlantic finfish. U. S. National Marine Fisheries Service. Fishery Bulletin 80: 745-759.

Montes-Hernández, E. 2006. Ictiofauna del arrecife rocoso de los Frailes, Baja California Sur, México. Informe Final de Servicio Social. Universidad Autónoma Metropolitana, Unidad Xochimilco. México, D.F., México.

Morris, R.H., D.P. Abbot \& E.C. Haderlie. 1980. Intertidal invertebrates of California. Stanford University, Stanford. Palo Alto, California, EEUU.
Pinkas L., M. Oliphant \& L.K. Iverson. 1971. Food habits of albacore, bluefin tuna, and bonito in California waters. California of Department Fish and Game, Fishery Bulletin152:105.

Reyes-Bonilla, H. 1997. Cabo Pulmo Reef: a new marine reserve in the Gulf of California. Conserv. Biol. 11:827.

Reyes-Bonilla, H. \& L.E. Calderón-Aguilera. 1999. Population density, distribution and consumption rates of three corallivores at Cabo Pulmo Reef, Gulf of California, México. Marine Ecol. 20:347-357.

Reyes-Nivia, M.C., J. Garzón-Ferreira \& A. RodríguezRamírez. 2004. Depredación de coral vivo por peces en el Parque Nacional Natural Tayrona, Caribe colombiano. Rev. Biol. Trop. 52:883-895.

Robertson, D.R. \& G.R. Allen. 2002. Shore fishes of the Tropical Eastern Pacific: an information system. CD-ROM. Smithsonian Tropical Research Institute, Balboa, Panama.

Robertson, D. R., J.S. Grove. y J.E. McCosker. (2004). Tropical transpacific shore fishes. Pacific Science. 58: 507-565.

Smith, P.E. \& M.T. Zaret. 1982. Bias in estimating niche overlap. Ecology 12: 48-1253.

Thomson, D.A., L.T. Findley \& A.N. Kerstitch. 2000. Reef fishes of the Sea of Cortez. The rocky-shore fishes of the Gulf of California. University of Texas, Texas, EEUU.

Zar, J.H. 1999. Biostatical analysis. Prentince-Hall, Englewood Clifs. Nueva Jersey, EEUU. 
